\title{
Correction: Behandlungstechnik in Katathym Imaginativer Psychotherapie, Hypnosepsychotherapie und Autogener Psychotherapie: Die Therapeutenversion der „Prozessskala für imaginations- und trancebasierte tiefenpsychologische Methoden" (PITT)
}

\author{
Christian Sell · Eveline Schöpfer-Mader · Bernhard Brömmel · Heidi Möller
}

Online publiziert: 24. November 2017

(c) Der/die Autor(en) 2017. Dieser Artikel ist eine Open-Access-Publikation.

\section{Correction: \\ Psychotherapie Forum 2017 \\ https://doi.org/10.1007/s00729-017-0102-2}

Der Artikel „Behandlungstechnik in Katathym Imaginativer Psychotherapie, Hypnosepsychotherapie und Autogener Psychotherapie: Die Therapeutenversion der ,Prozessskala für imaginations- und trancebasierte tiefenpsychologische Methoden' (PITT)“, von Christian Sell, Eveline Schöpfer-Mader, Bernhard Brömmel und Heidi Möller war fehlerhaft. Leider haben wir als Autorenteam erst nach Abschluss des Publikationsprozesses realisiert, dass die Abkürzung PITT im Feld der Psychotherapie bereits für die „Psychodynamisch Imaginative Trauma-Therapie“ nach Luise
Reddemann etabliert sowie markengeschützt ist. Wir bedauern diese Überschneidung und werden in der Folge die von uns entwickelte „Prozessskala für imaginations- und trancebasierte tiefenpsychologische Methoden“ von nun an mit PIT abkürzen.

Der Original-Beitrag wurde korrigiert.

Open Access Dieser Artikel wird unter der Creative Commons Namensnennung 4.0 International Lizenz (http:// creativecommons.org/licenses/by/4.0/deed.de) veröffentlicht, welche die Nutzung, Vervielfältigung, Bearbeitung, Verbreitung und Wiedergabe in jeglichem Medium und Format erlaubt, sofern Sie den/die ursprünglichen Autor(en) und die Quelle ordnungsgemäß nennen, einen Link zur Creative Commons Lizenz beifügen und angeben, ob Änderungen vorgenommen wurden.

Die Online-Version des Originalartikels ist unter https://doi. org/10.1007/s00729-017-0102-2 zu finden.

\author{
C. Sell $(\bowtie) \cdot H$. Möller \\ Institut für Psychologie, Universität Kassel, Holländische Str. \\ 36-38, 34127 Kassel, Deutschland \\ csell@uni-kassel.de \\ E. Schöpfer-Mader \\ Herzog-Friedrich-Straße 19, 6020 Innsbruck, Österreich \\ B. Brömmel \\ Penzinger Straße 106/2, 1140 Wien, Österreich \\ E. Schöpfer-Mader $\cdot$ B. Brömmel \\ Österreichische Gesellschaft für angewandte \\ Tiefenpsychologie und allgemeine Psychotherapie \\ (ÖGATAP), 1070 Wien, Österreich
}

